\title{
Characterization and applications of a tunable, laser-based, MeV-class Compton-scattering $\gamma$-ray source
}

\author{
F. Albert, S. G. Anderson, D. J. Gibson, C. A. Hagmann, M. S. Johnson, M. Messerly, V. Semenov, M. Y. Shverdin, \\ B. Rusnak, A. M. Tremaine, F. V. Hartemann, C. W. Siders, D. P. McNabb, and C. P. J. Barty \\ Lawrence Livermore National Laboratory, 7000 East Avenue, Livermore, California 94550, USA
}

(Received 27 October 2009; published 27 July 2010)

\begin{abstract}
A high peak brilliance, laser-based Compton-scattering $\gamma$-ray source, capable of producing quasimonoenergetic photons with energies ranging from 0.1 to $0.9 \mathrm{MeV}$ has been recently developed and used to perform nuclear resonance fluorescence (NRF) experiments. Techniques for characterization of $\gamma$-ray beam parameters are presented. The key source parameters are the size $\left(0.01 \mathrm{~mm}^{2}\right)$, horizontal and vertical divergence $\left(6 \times 10 \mathrm{mrad}^{2}\right)$, duration $(16 \mathrm{ps})$, and spectrum and intensity $\left(10^{5}\right.$ photons $/$ shot $)$. These parameters are summarized by the peak brilliance, $1.5 \times 10^{15}$ photons $/ \mathrm{mm}^{2} / \mathrm{mrad}^{2} / \mathrm{s} / 0.1 \%$ bandwidth, measured at $478 \mathrm{keV}$. Additional measurements of the flux as a function of the timing difference between the drive laser pulse and the relativistic photoelectron bunch, $\gamma$-ray beam profile, and background evaluations are presented. These results are systematically compared to theoretical models and computer simulations. NRF measurements performed on ${ }^{7} \mathrm{Li}$ in $\mathrm{LiH}$ demonstrate the potential of Compton-scattering photon sources to accurately detect isotopes in situ.
\end{abstract}

DOI: 10.1103/PhysRevSTAB.13.070704

PACS numbers: 41.60.- m, 07.85.Fv, 52.38.Ph

\section{INTRODUCTION}

Over the past two decades, considerable technological improvements in the field of high intensity lasers, highbrightness electron linacs, and x-ray diagnostics have contributed to the maturation of a novel type of light sources based on Compton scattering, where incident laser photons are scattered by a relativistic electron beam to generate tunable, highly collimated light pulses with picosecond or femtosecond durations, and relatively narrow spectral bandwidth [1]. Concurrently, an increasing number of important applications are being explored, both experimentally and via detailed computer simulations. At photon energies below $100 \mathrm{keV}$, advanced biomedical imaging techniques, including ultrafast $\mathrm{x}$-ray protein crystallography [2], phase contrast imaging [3], and $K$-edge imaging [4], are under consideration by a number of groups worldwide. Although synchrotron light sources [5] and x-ray free-electron lasers such as LCLS [6] or the European $X F E L$ [7] can produce $x$ rays at higher brightness in this energy range, Compton-scattering light sources are attractive because of their compact footprint. At $\gamma$-ray photon energies relevant for nuclear processes and applications, these new radiation sources will produce the highest peak brilliance. The applications include nuclear resonance fluorescence (NRF) [8], picosecond positron beams [9], and photo fission. $\mathrm{HI} \gamma \mathrm{S}$ [10], a large 2-86 MeV highenergy $\gamma$-ray facility producing polarized photons via intracavity Compton backscattering from a free-electron laser, has been used as a research tool to assign the parity of excited states in nuclei [11].

In this paper we describe the characterization techniques and applications of a monoenergetic gamma-ray (MEGaray) source: At LLNL we have optimized the laser Compton process to produce high brilliance photon beams in the $0.1-0.9 \mathrm{MeV}$ spectral range. The paper is organized as follows: in Sec. II, properties of Compton backscattering sources as well as relevant theoretical considerations are presented, which also forms the basis of the computer codes used to analyze the data. Section III presents a general overview of the experimental system and the detection techniques, and Sec. IV reviews the full characteristics of the photon beam, such as its spatial and spectral information. Comparisons with theory are provided throughout the analysis of our data. Finally, Sec. V presents the results of NRF in ${ }^{7} \mathrm{Li}$ as an application of this source before concluding in Sec. VI.

\section{PROPERTIES OF COMPTON-SCATTERING SOURCES}

\section{A. Basic properties}

The properties of Compton-scattering sources, which have been extensively studied [12-16], rely on energymomentum conservation. With this feature, one can derive the exact relativistic Doppler shifted energy:

$$
E_{x}=\frac{\gamma-\sqrt{\gamma^{2}-1} \cos \phi}{\gamma-\sqrt{\gamma^{2}-1} \cos \theta+\lambda k_{0}(1-\cos \theta \cos \phi+\cos \psi \sin \theta \sin \phi)} E_{L}
$$


where $\gamma$ is the electron relativistic factor, $\phi$ is the angle between the incident laser and electron beams, $\theta$ the angle between the scattered photon and incident electron, $\psi$ the angle between the incident and scattered photon, $k_{0}=$ $2 \pi / \lambda$ the laser wave number, where $\lambda_{c}=3.8616 \times$ $10^{-13} \mathrm{~m}$ is the reduced Compton wavelength, and finally $E_{L}$ is the laser energy. In this case we have assumed the fact that $\beta=v / c \simeq 1$, where $v$ is the electron velocity, which is typical in our experiments. In the case of a head-on collision (the angle between the incident photons and electrons is $\phi=180^{\circ}$ ), and on-axis observation (we observe the scattered photons at an angle $\theta=0^{\circ}$ with respect to the axis defined by the incident electron beam), and if the observation occurs in the incidence plane defined by the incident electron and photon beams $(\psi=0)$, then the scattered energy roughly scales as $4 \gamma^{2} E_{L}$. Indeed, The electron recoil, in this case $4 \gamma k_{0} \lambda_{c}$, is a few $10^{-3}$ for our experimental parameters $\left(\gamma \simeq 200, k_{0} \simeq 10^{7}\right)$ and can therefore be neglected. This makes Compton-scattering sources very attractive because one can obtain high-energy $(\mathrm{MeV})$ scattered photons with relatively modest electron beam energies, making the source rather compact compared to machines like third generation synchrotrons [5]. An important feature of Compton-scattering sources is that the Compton-scattering cross section is very small $(\sigma=$ $6.65 \times 10^{-25} \mathrm{~cm}^{2}$ ), so a high density of electrons and photons (and thus very high-quality beams) is required at the interaction point. In the case where the laser focal spot and electron bunch focus have similar size, $w_{0}$, the number of $\mathrm{x}$ rays produced can be approximated by $N_{x}=$ $\left(\sigma / \pi w_{0}^{2}\right) N_{L} N_{e}$, where $N_{L}$ and $N_{e}$ are, respectively, the number of laser photons and the number of electrons in the bunch, assumed to be fixed. In the case of a beam with conserved normalized emittance (true in a linear accelerator), and with negligible recoil, the electron beam focal spot size scales as $1 / \gamma$, and thus the $\mathrm{x}$-ray yield varies as $\gamma^{2}$. While typical synchrotrons provide the highest brightness in the 10-100 keV range, Compton-scattering sources become a more efficient option at higher energies. Just like synchrotrons, Compton-scattering sources are attractive because they are highly collimated and have a good energy-angle correlation.

\section{B. Modeling \\ 1. Normalized spectrum}

The computer code used to analyze the data considers Compton scattering by electrons with a given phase space distribution interacting with a Gaussian-paraxial electromagnetic wave, but neglecting wave front curvature. This is adequate for laser foci with sufficiently large $(f / D>$ 10) $F$ numbers (diffraction limited spot size of $1.22 \lambda f / D$, where $f$ is the focal length, $D$ the beam diameter, and $\lambda$ the laser wavelength). The key quantities used in our analysis and a detailed description of the formalism used here can be found in Ref. [17]. We first calculate the Comptonscattering frequency by utilizing the energy-momentum conservation law:

$$
\kappa-\lambda=\lambda_{c}\left(k_{\mu} q^{\mu}\right)
$$

where $\lambda_{c}=\hbar / m_{0} c$ is the reduced Compton wavelength of the electron, $\kappa$ and $\lambda$ are the incident and scattered light cone variables, $k_{\mu}=(k, 0,0, k)$ is the incident laser pulse 4-wave vector and $q_{\mu}$ is the scattered 4-wave vector. By solving this equation for $q$, one obtains $q_{c}$, the wave number for the scattered radiation:

$$
q_{c}=\frac{k\left(\gamma-u_{z}\right)}{\gamma-u_{z} \cos \theta+k \lambda_{c}\left(1-u_{x} \sin \theta \cos \phi-u_{y} \sin \theta \sin \phi-u_{z} \cos \theta\right)},
$$

where $\theta$ and $\phi$ refer to the classical spherical coordinates (in the case of a counterpropagating scheme $\theta=\pi$ and $\phi=0)$ and where $u_{\mu}=\left(\gamma=\sqrt{1+u_{x}^{2}+u_{y}^{2}+u_{z}^{2}}, u_{x}\right.$, $\left.u_{y}, u_{z}\right)$ is the electron 4-velocity and $\gamma$ its relativistic factor. From there, one can generate a random normal distribution of particles with velocities $u_{x}, u_{y}$ and relativistic factor $\gamma$ and standard deviations $\Delta u_{x}=j \epsilon_{x} / \sigma_{x}, \Delta u_{y}=j \epsilon_{y} / \sigma_{y}$, and $\Delta_{\gamma}$, respectively. The quantities $\epsilon$ and $\sigma$ refer to the electron beam normalized emittance and spot size and $j$ to the jitter; $j=1$ means that there is no jitter, $j=2$ means that the electron beam angular spread is multiplied by two for a given spot size. Figure 1 gives an example of a normalized spectrum obtained with this code.

\section{Dose}

To calculate spectral distribution yielded by the interaction, one has to take into account several other parameters.
The most useful expression to describe the source is typically the local differential brightness, which can be derived from the local number of photons scattered per unit time and volume [18]:

$$
\frac{d^{12} N}{d^{4} x d \Omega d q d^{3} u d^{3} k}=\frac{d \sigma}{d \Omega} \delta\left(q-q_{c}\right) \frac{d^{3} n_{e}}{d^{3} u} \frac{d^{3} n_{\lambda}}{d^{3} k} \frac{u_{\mu} k^{\mu}}{\gamma k},
$$

where $d^{3} n_{e} / d^{3} u$ and $d^{3} n_{\lambda} / d^{3} k$ represent the electron beam phase space density and laser pulse momentum space density.

We start with the differential scattering cross section $d \sigma / d \Omega$ described by the Lorentz-boosted Klein-Nishina formula, as derived by Bhatt et al. [19], in which we only use the spin-independent component: 


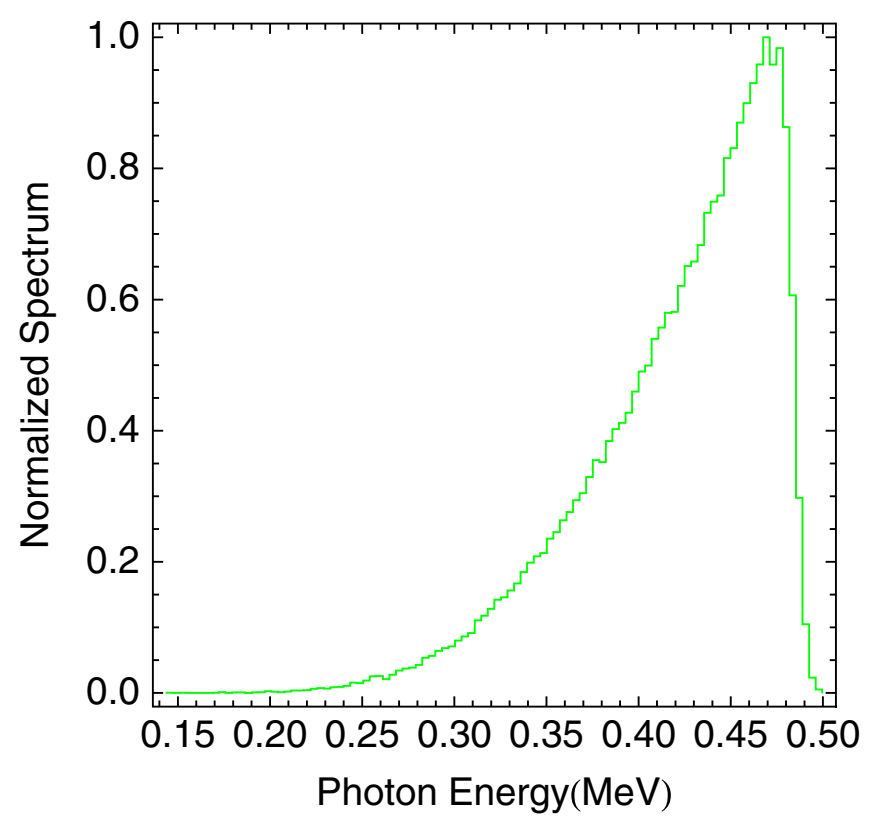

FIG. 1. (Color) Example of a spectrum simulated with MATHEMATICA, using 100000 particles and 100 bins for an electron beam energy of $116 \mathrm{MeV}$ and a laser wavelength of $532 \mathrm{~nm}$ (energy $2.33 \mathrm{eV}$ ). The other parameters are $j=2, \epsilon_{x}=$ $5 \mathrm{~mm} \mathrm{mrad}, \epsilon_{y}=6 \mathrm{~mm} \mathrm{mrad}, \sigma_{x}=35 \mu \mathrm{m}$, and $\sigma_{y}=40 \mu \mathrm{m}$.

$$
\begin{aligned}
\frac{d \sigma}{d \Omega}= & \frac{1}{2}\left(\alpha \lambda_{c}\right)^{2}\left(\frac{q}{\kappa}\right)^{2}\left[\frac{1}{2}\left(\frac{\kappa}{\lambda}+\frac{\lambda}{\kappa}\right)-1\right. \\
& \left.+2\left(\epsilon_{\mu} \pi^{\mu}-\frac{\left(\epsilon_{\mu} u^{\mu}\right)\left(\pi_{\nu} v^{\nu}\right)}{\kappa \lambda_{c}}+\frac{\left(\epsilon_{\mu} v^{\mu}\right)\left(\pi_{\nu} u^{\nu}\right)}{\lambda \lambda_{c}}\right)^{2}\right]
\end{aligned}
$$

where $\alpha$ is the fine structure constant, $\epsilon_{\mu}=(0,1,0,0)$ corresponds to a linearly polarized incident radiation, and $\pi_{\mu}$ is the scattered 4-polarization. $v_{\mu}=u_{\mu}+\lambda_{c}\left(k_{\mu}-\right.$ $\left.q_{\mu}\right)$ is the 4-velocity after the scattering event.

In the case of a single electron following a trajectory $\mathbf{r}(\tau)$, where $\tau$ is its proper time, the phase space density is given by a product of Dirac delta distributions:

$$
\begin{aligned}
\frac{d^{3} n_{e}}{d^{3} u} & =\delta[\mathbf{x}-\mathbf{r}(\tau)] \delta[\mathbf{u}-\mathbf{u}(\tau)] \\
& =\delta[\mathbf{x}-\mathbf{r}(\tau)] \delta\left[\mathbf{u}-\frac{d \mathbf{r}(\tau)}{c d \tau}\right]
\end{aligned}
$$

and thus, after integration over all the electron phase space, the integrated brightness (over the whole surface of the source) reads

$$
\begin{aligned}
\frac{d^{3} N}{c d t d \Omega d q}= & \iiint_{\mathbb{R}^{3}} \frac{d \sigma}{d \Omega} \delta\left(q-q_{c}\right) \frac{d^{3} n_{\lambda}}{d^{3} k}[\mathbf{r}(\tau), \tau] \\
& \times \frac{u_{\mu}(\tau)}{\gamma(\tau)} \frac{k^{\mu}}{k} d^{3} k .
\end{aligned}
$$

In the case of an uncorrelated incident photon phase space, corresponding to the Fourier transform limit, the phase space density takes the form of a product,

$$
\frac{d^{3} n_{\lambda}}{d^{3} k}=n_{\lambda}\left(x_{\mu}\right) \tilde{n}_{\lambda}\left(k_{\nu}\right)
$$

the number of photons scattered per unit wave number and solid angle is then given by

$$
\begin{aligned}
\frac{d^{2} N}{d \Omega d q}= & \int_{-\infty}^{\infty} n_{\lambda}[\mathbf{r}(\tau), \tau] c d t \iiint_{\mathbb{R}^{3}} \frac{d \sigma}{d \Omega} \delta\left(q-q_{c}\right) \tilde{n}_{\lambda}\left(k_{\nu}\right) \\
& \times \frac{\kappa}{\gamma k} d^{3} k .
\end{aligned}
$$

In our analysis, we consider the case of a plane wave in the Fourier domain:

$$
k_{x}=k_{y}=0, \quad k_{z}=k, \quad \tilde{n}_{\lambda}=\frac{\exp \left[-\left(\frac{k-k_{0}}{\Delta k}\right)^{2}\right]}{\sqrt{\pi} \Delta k} .
$$

The integral over $k$ is easily performed by using the fact that

$$
\delta[f(x)]=\sum \frac{\delta\left(x-x_{n}\right)}{\left|f^{\prime}\left(x_{n}\right)\right|},
$$

where $x_{n}$ represents the poles of the function $f$. Applying this rule to our case, we first look for the poles $k_{p}$ of $q_{c}$ by solving for $q_{c}(k)=0$, and using the above in (9), we find

$$
\begin{aligned}
\frac{d^{2} N}{d \Omega d q}= & \frac{1}{\sqrt{\pi} \Delta k}\left[\frac{d \sigma}{d \Omega} \frac{\kappa}{\gamma k} \frac{e^{-\left(k-k_{0}\right)^{2} / \Delta k^{2}}}{\left|\partial_{k} q_{c}(k)\right|}\right]_{k=k_{p}} \\
& \times \int_{-\infty}^{\infty} n_{\lambda}[\mathbf{r}(\tau), \tau] c d t .
\end{aligned}
$$

For a Gaussian laser pulse, the incident photon density can be modeled analytically within the paraxial approximation, and in the case of a cylindrical focus:

$$
\begin{aligned}
n_{\lambda}(\mathbf{x}, t)= & \frac{N_{\lambda}}{\sqrt{\pi / 2} w_{0}^{2} c \Delta t 3} \frac{1}{1+\left(z / z_{0}\right)^{2}} \\
& \times \exp \left[-2\left(\frac{t-z / c}{\Delta t}\right)^{2}-2 \frac{r^{2}}{w_{0}^{2}\left[1+\left(z / z_{0}\right)^{2}\right]}\right],
\end{aligned}
$$

where $N_{\lambda}$ is the total number of photons in the laser pulse, $\Delta t$ the pulse duration, $w_{0}$ the $1 / e^{2}$ focal radius, and $z_{0}=$ $\pi w_{0}^{2} / \lambda_{0}$ is the Rayleigh range. To evaluate the integral in (12), we replace the spatial coordinates by the ballistic electron trajectory:

$$
\begin{array}{ll}
x(t)=x_{0}+\frac{u_{x}}{\gamma} c t, & y(t)=y_{0}+\frac{u_{y}}{\gamma} c t, \\
z(t)=z_{0}+\frac{u_{z}}{\gamma} c t, & r^{2}(t)=x^{2}(t)+y^{2}(t),
\end{array}
$$


where we can divide $x, y$, and $r$ by $w_{0}$ and $z$ and $c t$ by $z_{0}$ to obtain the normalized quantities $\bar{x}, \bar{y}, \bar{z}, \bar{r}$, and $\bar{t}$. One finally obtains the expression

$$
\begin{aligned}
\frac{d^{2} N}{d \Omega d q}= & \frac{1}{\sqrt{\pi} \Delta k}\left[\frac{d \sigma}{d \Omega} \frac{\kappa}{\gamma k} \frac{e^{-\left(k-k_{0}\right)^{2} / \Delta k^{2}}}{\left|\partial_{k} q_{c}(k)\right|}\right]_{k=k_{p}} \frac{N_{\lambda}}{\sqrt{\pi / 2} w_{0}^{2} c \Delta t 3} \\
& \times \int_{-\infty}^{\infty} \frac{1}{1+\bar{z}^{2}} \\
& \times \exp \left[-2\left(\frac{z_{0}}{c \Delta t}\right)^{2}(\bar{t}-\bar{z})^{2}-2 \frac{\bar{r}^{2}}{1+\bar{z}^{2}}\right] d \bar{t} .
\end{aligned}
$$

We finally note that when evaluating (15) it is sufficient to calculate the integral within an interval of $\sim 10 \Delta t$ as we assume a Gaussian temporal pulse profile.

\section{Integrated dose}

Calculating the total integrated number of $\gamma$-ray photons is somewhat simpler. As derived in Ref. [18], the temporal behavior of the $\gamma$-ray pulse can be described in Cartesian coordinates:

$$
\frac{d^{3} N}{2 \pi r d r d z c d t}=\sigma\left(1+\beta_{0}\right) n_{\lambda}(r, z, t) n_{e}(r, z, t)
$$

where $n_{e}(r, z, t)$ is the incident electron beam density and $\sigma=8 \pi r_{0}^{2} / 3$ is the Compton-scattering cross section. To evaluate the dose yielded by only one macroparticle along its trajectory $\left(r_{e}, z_{e}\right)$, one can replace $n_{e}(r, z, t)$ by $\delta(r=$ $\left.r_{e}, z=z_{e}, t\right)$ to end with

$$
\begin{aligned}
\frac{d^{3} N_{i}}{c d t} & =\sigma\left(1+\beta_{0}\right) n_{\lambda}(r, z, t) \delta\left(r=r_{e}, z=z_{e}, t\right) \\
& =\sigma\left(1+\beta_{0}\right) n_{\lambda}\left(r=r_{e}, z=z_{e}, t\right) .
\end{aligned}
$$

In other words,

TABLE I. Laser and electron beam parameters used for the integrated dose calculation. $20 \%$ compressed means that only $20 \%$ of the total laser energy is compressed within the duration of the pulse.

\begin{tabular}{lc}
\hline \hline Parameter & Specification \\
\hline Laser pulse duration & $20 \mathrm{ps}(\mathrm{FWHM})$ \\
Laser wavelength & $532 \mathrm{~nm}$ \\
Laser spot size & $34 \mu \mathrm{m}(\mathrm{rms}), 20 \%$ energy in spot \\
Laser energy & $150 \mathrm{~mJ}, 20 \%$ compressed \\
Electron energy & $116 \mathrm{MeV}$ \\
Electron beam spot size & $40 \mu \mathrm{m}(\mathrm{rms})$ \\
Electron bunch length & $20 \mathrm{ps}(\mathrm{FWHM})$ \\
Electron beam charge & $0.5 \mathrm{nC}$ \\
Normalized emittance & $6 \mathrm{~mm} \mathrm{mrad}$ \\
jitter factor & 2 \\
\hline \hline
\end{tabular}

$$
\begin{aligned}
N_{i}= & \sigma\left(1+\beta_{0}\right) \frac{N_{\lambda}}{\sqrt{\pi / 2} w_{0}^{2} \Delta t 3} \int_{-\infty}^{\infty} \frac{1}{1+\bar{z}^{2}} \\
& \times \exp \left[-2\left(\frac{z_{0}}{c \Delta t}\right)^{2}(\bar{t}-\bar{z})^{2}-2 \frac{\bar{r}^{2}}{1+\bar{z}^{2}}\right] d \bar{t},
\end{aligned}
$$

where normalized quantities have been used again. To evaluate the integral in (18), we can use again the electron's ballistic trajectories, as defined in (14).

The total dose is then simply obtained by summing over all the macroparticles, and by taking into account the ratio $N_{e} / n$ between the total number of electrons in the beam and macroparticles in the code. By using this model, we obtain an integrated (over all solid angles) dose of $N=$ $1.32 \times 10^{5}$ photons, for the laser and electron beam parameters summarized in Table I.

\section{EXPERIMENTAL METHODS}

\section{A. Setup overview}

The experiments have been performed at the LLNL $100 \mathrm{MeV}$ linac facility, which is located in a well shielded environment, 10 meters below ground, where previous Compton scattering experiments have been performed [20-22]. An overview of the experimental facility is presented in Fig. 2. There were three main caves in which the experiments were made: the outer-detector cave, where the interaction laser system (ILS) was located; the accelerator cave, containing the photocathode and the photoinjector drive laser (PDL), the linac, and the interaction point; and

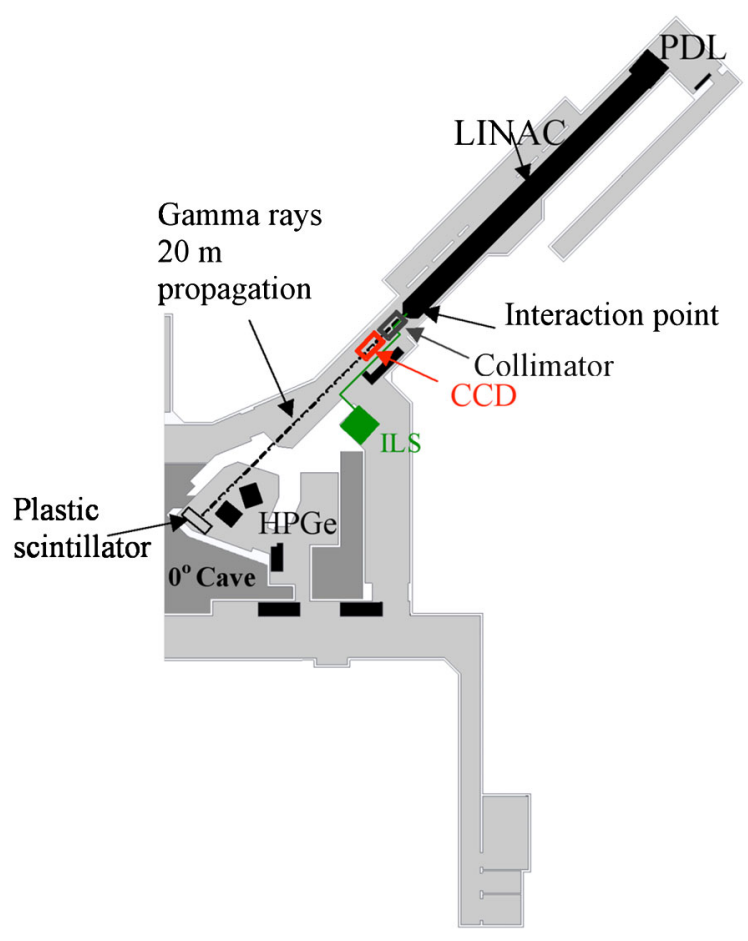

FIG. 2. (Color) Overview of the experimental facility with the outer-detector (laser) cave, the accelerator cave, and the $0^{\circ}$ cave. 
finally the $0^{\circ}$ cave, located 20 meters away from the interaction point, on the other side of a thick concrete wall where the $\gamma$-ray diagnostics, including germanium detectors, were set up.

At first light, the $\gamma$ rays were detected on-axis by an intensified CCD mounted on a translation stage. Once the $\gamma$-ray beam profile was seen on the camera, we translated a $6 \mathrm{~mm}$ diameter aperture lead collimator (located 3 meters away from the interaction point) in the beam, and then the camera out, to allow the $\gamma$ rays to propagate in air to the $0^{\circ}$ cave. In the $0^{\circ}$ cave, the $\gamma$ rays were first detected by a large plastic paddle-shaped scintillator coupled to a $1.3 \mathrm{kV}$ biased photomultiplier. Once $\gamma$ rays correlated to the electron/laser interaction were seen on this detector, spectral measurements using the germanium detectors could be performed.

For clarity and comprehension, Sec. III B gives a short description of the three main parts of the setup used to produce $\gamma$ rays: the laser system, the linac, and the interaction region. For a more exhaustive description of the full system, we refer the reader to Gibson et al. [23], and references therein. Then Sec. IIIC describes the experimental methods used to characterize the beam.

\section{B. $\gamma$-ray light source system}

The ILS delivers $750 \mathrm{~mJ}$ of energy in $16 \mathrm{ps}, 0.2 \mathrm{~nm}$ bandwidth pulses at $10 \mathrm{~Hz}$. It uses chirped pulse amplification (CPA) [24] in a flashlamp pumped commercial Nd: YAG bulk amplifier fed by a laser oscillator. The key enabling technology is the use of a novel hyperdispersion compressor matched to a chirped fiber Bragg grating stretcher in the front end. The beam after the compressor, initially at $1064 \mathrm{~nm}$, can be frequency doubled or tripled using large aperture deuterated potassium phosphate (DKDP) crystals. For the work here, the $1064 \mathrm{~nm}$ pulse of the ILS is frequency doubled using a DKDP crystal, which yields $146 \mathrm{~mJ}$ of $532 \mathrm{~nm}$ light.

The accelerator that provides the electrons starts with a 1.6 cell photocathode gun with a sputtered $\mathrm{Mg}$ cathode. This gun is driven by the PDL which shares the oscillator with the ILS to ensure synchronization and provides a $30 \mu \mathrm{J}$ 15-ps flattop spatial and temporal profile at $263.25 \mathrm{~nm}$. This gun produces a charge of $800 \mathrm{pC}$ during normal operation that is then accelerated in five $2.5 \mathrm{~m}$ SLAC-type $s$-band traveling wave sections. The normalized emittance at the end of the beam line is $5-6 \mathrm{~mm} \mathrm{mrad}$ and it has been measured at low energy using a pepper-pot mask and at high energy with the quadrupole scan technique. After colliding with the laser pulse, the electrons are swept off axis by a $20^{\circ}$ dipole magnet to separate the electron bunch from the $\gamma$ rays and to measure the energy spectra of the electron pulses.

As it is the configuration that provides the brightest output (all the electrons from the bunch are illuminated by the ILS), the laser and electron beams collide at $180^{\circ}$.
The laser beam is focused to $34 \mu \mathrm{m}$ (rms) and the electron beam is focused using a set of quadrupole magnets with fields of up to $15 \mathrm{~T} / \mathrm{m}$. At the focal point, spatial and temporal overlap of the two beams is performed by using an optically polished nickel cube mounted on a three-axis translation stage, with faces that are vertically oriented perpendicular to the beam line, and horizontally oriented at $45^{\circ}$ with respect to the beam line. When the electron beam hits the cube, it produces optical transition radiation, and the laser beam is also reflected from the surface. Hence, the position of the two beams at the focal point can be imaged by a CCD camera on one side of the cube for the spatial overlap and by a $100 \mu \mathrm{m}$ slit streak camera providing a 2 ps resolution on the other side for the temporal overlap.

\section{Methods and diagnostics for $\gamma$-ray beam characterization}

\section{Spatial and temporal measurements}

The $\gamma$ rays are detected on axis by a system which is comprised of a 16 bit intensified charge coupled device (ICCD) Andor camera and a 3:1 optical fiber reducer coupled to the camera. The ICCD chip is an array of $1024 \times 102413 \mu \mathrm{m}$ size pixels coupled to a 1:1 optical fiber bundle, which, with the reducer demagnification, provides a $4 \times 4 \mathrm{~cm}^{2}$ detection surface. The fiber reducer is coupled to a $140 \mu \mathrm{m}$ cesium iodine scintillator doped with thallium [CsI(Tl)], which isotropically converts the $\gamma$ rays into visible green light at a rate of 54 photons $/ \mathrm{keV}$. The scintillator is protected with a $0.5 \mathrm{~mm}$ thick beryllium (Be) window to block any laser light remaining and to cut detected noise from low energy $x$ rays scattering in the accelerator cave. The system was calibrated by using a ${ }^{137} \mathrm{Cs}$ radioisotope source delivering $2.85 \times 10^{5}$ photons/s at $662 \mathrm{keV}$. The measurements indicate 70 counts/photon at this energy (one gamma-ray photon yields 70 counts on the CCD). From that point, the number of counts per $\gamma$-ray photon produced by the detection system can be extrapolated by using the $\gamma$-ray absorption curve in $\mathrm{CsI}(\mathrm{Tl})$, which can be found in the NIST X-COM database [25]:

$$
C(E)=\frac{E}{E_{0}} C\left(E_{0}\right) \frac{1-e^{-\rho \mu(E) \Delta}}{1-e^{-\rho \mu\left(E_{0}\right) \Delta}},
$$

where $\Delta=140 \mu \mathrm{m}$ is the scintillator thickness, $E_{0}=$ $662 \mathrm{keV}$ is the energy of the calibration source, $r=$ $4.51 \mathrm{~g} / \mathrm{cm}^{3}$ is the density of $\operatorname{CsI}(\mathrm{Tl})$, and $\mu(E)$ is the mass absorption coefficient, in square centimeters per gram. Because we made the calibration at a different energy $(662 \mathrm{keV})$ than that of our source $(478 \mathrm{keV})$, Eq. (19) basically takes into account the fact that the detector response is not constant with energy. 


\section{Spectral measurements}

Several techniques are currently available to measure $\mathrm{x}$ ray spectra, including Bragg diffraction crystals, filters relying on x-ray attenuation in materials, and photoncounting methods with scintillators or x-ray diodes. As the lattice spacing for a diffraction crystal has to be on the order of the wavelength of the diffracted radiation to obtain a good resolution and efficiency, no crystal allows us to use this technique at energies of a few $100 \mathrm{keV}$ and above. Using filters in our case is impractical because high $Z$ and very thick materials would be needed, and the resolution of the measurement would be poor. For our spectral experiments we decided to use a detector operating in a statistical single photon-counting mode.

In the present geometry, the $\gamma$-ray detector would have to be directly in the high-energy bremsstrahlung produced by the dark current of the linac if we want to measure the spectrum on axis. This yields background levels that are incompatible with single photon counting. To avoid this problem, we chose a geometry where the $\gamma$ rays are detected by a high purity germanium detector (HPGe) at an angle $\theta=48^{\circ}$ with respect to the main beam axis, after being scattered off a $1 / 8$ inch thick aluminum (Al) plate located 20 meters away from the interaction point, in a room where the system is shielded by concrete walls. With this layout, as depicted on Fig. 3, the scattered $\gamma$ rays of energy $E_{\gamma}$ are related to the incident $\gamma$ rays of energy $E_{\gamma}$ by the Compton-scattering relation:

$$
E_{\gamma}^{\prime}=\frac{E_{\gamma}}{1+\frac{E_{\gamma}}{E_{0}}(1-\cos \theta)},
$$

where $E_{0}=0.511 \mathrm{MeV}$ is the electron rest energy.

This method has two advantages: the detector is placed far from the on-axis bremsstrahlung background and the $\mathrm{Al}$ plate preferentially scatters the lower energy $\gamma$ rays. For example, $7 \%$ of $500 \mathrm{keV}$ radiation is attenuated by the plate while only $2.5 \%$ of $5 \mathrm{MeV} \gamma$ rays are. The thickness

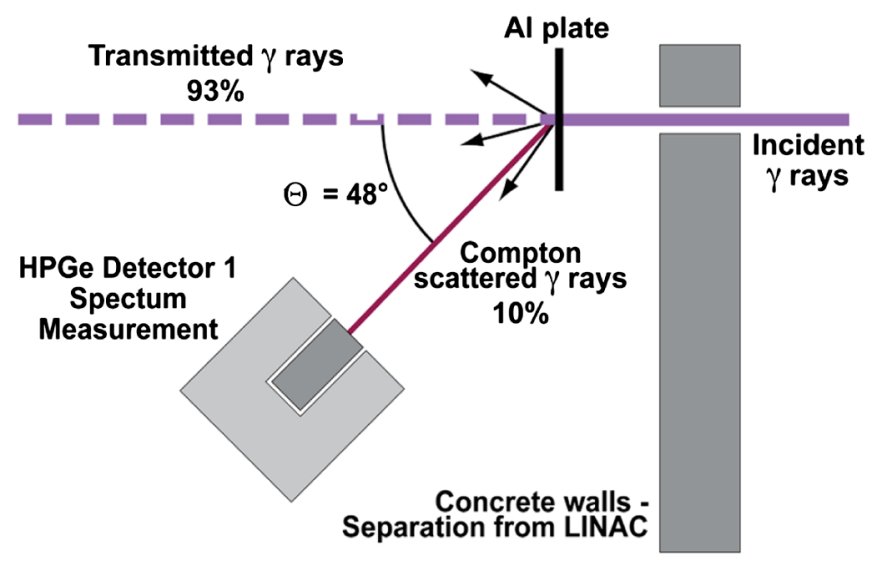

FIG. 3. (Color) Layout of the detection system. The $\gamma$ rays are detected by a HPGe detector after being Compton scattered off an $\mathrm{Al}$ plate at 48 degrees. of the Al plate was adjusted so that a count rate of 0.2 photon/shot was observed on the HPGe, which we judged as the best tradeoff between pileup and efficiency. The detector is made of a high purity germanium crystal which is $8 \mathrm{~cm}$ long and $6 \mathrm{~cm}$ in diameter. The resolution, measured with a ${ }^{137} \mathrm{Cs}$ radioisotope source, was found to be $2.8 \mathrm{keV}$ at $662 \mathrm{keV}(0.4 \%)$. The detector head is placed $150 \mathrm{~cm}$ away from the $\mathrm{Al}$ plate, which corresponds to an angle of $2.3^{\circ}$ subtended by the crystal. With a scattering angle of 48 degrees and a central energy of $478 \mathrm{keV}$ (scattered energy $365 \mathrm{keV}$ ), this means an uncertainty of $13 \mathrm{keV}$ on the spectrum measurement (relative uncertainty of $3.5 \%$ ). The resolution of our spectrum is thus limited by the geometry of our detection system. The output signal of the detector, biased at +4000 Volts, is shaped to a pulse with an amplitude ranging from 1 to 10 Volts and with a full width half maximum (FWHM) of several $\mu \mathrm{s}$. This pulse is then sent to a 8192-channel analog to digital converter (ADC) which retrieves the spectrum. The ADC was synchronized to the rf power of the linac with a $8 \mu \mathrm{s}$ gate, to ensure that only photon events related to the linac and Compton scattered $\gamma$ rays at $10 \mathrm{~Hz}$ where recorded. This was necessary because of the natural activation present in the room, at a rate of several $\mathrm{kHz}$, which makes the detection of the Compton scattered $\gamma$-rays spectrum impossible. The settings of the shaper and ADC allowed us to measure spectra in the $10 \mathrm{keV}-1.6 \mathrm{MeV}$ range with a dispersion of $0.2 \mathrm{keV} /$ channel.

\section{EXPERIMENTAL RESULTS}

This section presents a complete characterization of the source mainly when the electron beam is tuned at $116 \mathrm{MeV}$ and the laser at $532 \mathrm{~nm}$ (second harmonic generation of $1064 \mathrm{~nm}$ infrared light produced by the ILS). We have also produced $\gamma$ rays with laser light from first $(1064 \mathrm{~nm})$ and third $(355 \mathrm{~nm})$ harmonic generation, and the results are presented for completeness, although no exhaustive characterization has been made at these energies. On axis, if we have $116 \mathrm{MeV}$ electrons and $532 \mathrm{~nm}$ laser light, the scattered energy is $478 \mathrm{keV}$, according to (1). Our motivation for selecting this energy was to detect nuclear resonance fluorescence (NRF) in ${ }^{7} \mathrm{Li}$, which has a strong line at $478 \mathrm{keV}$. At this energy, the following features of the source have been studied: $\gamma$-ray beam profile and dose, source size, dose as a function of delay between the laser and the electron beam, on and off-axis spectrum, and tunability with the electron beam energy.

\section{A. Spatial and temporal properties of the source}

\section{Beam profile and dose}

The spatial beam profile was measured with the Andor camera using $15 \mathrm{~s}$ integration time $(150$ shots at $10 \mathrm{~Hz})$ to obtain good statistics. Figure 4 shows a typical $\gamma$-ray image recorded with those conditions $2 \mathrm{~m}$ away from the 

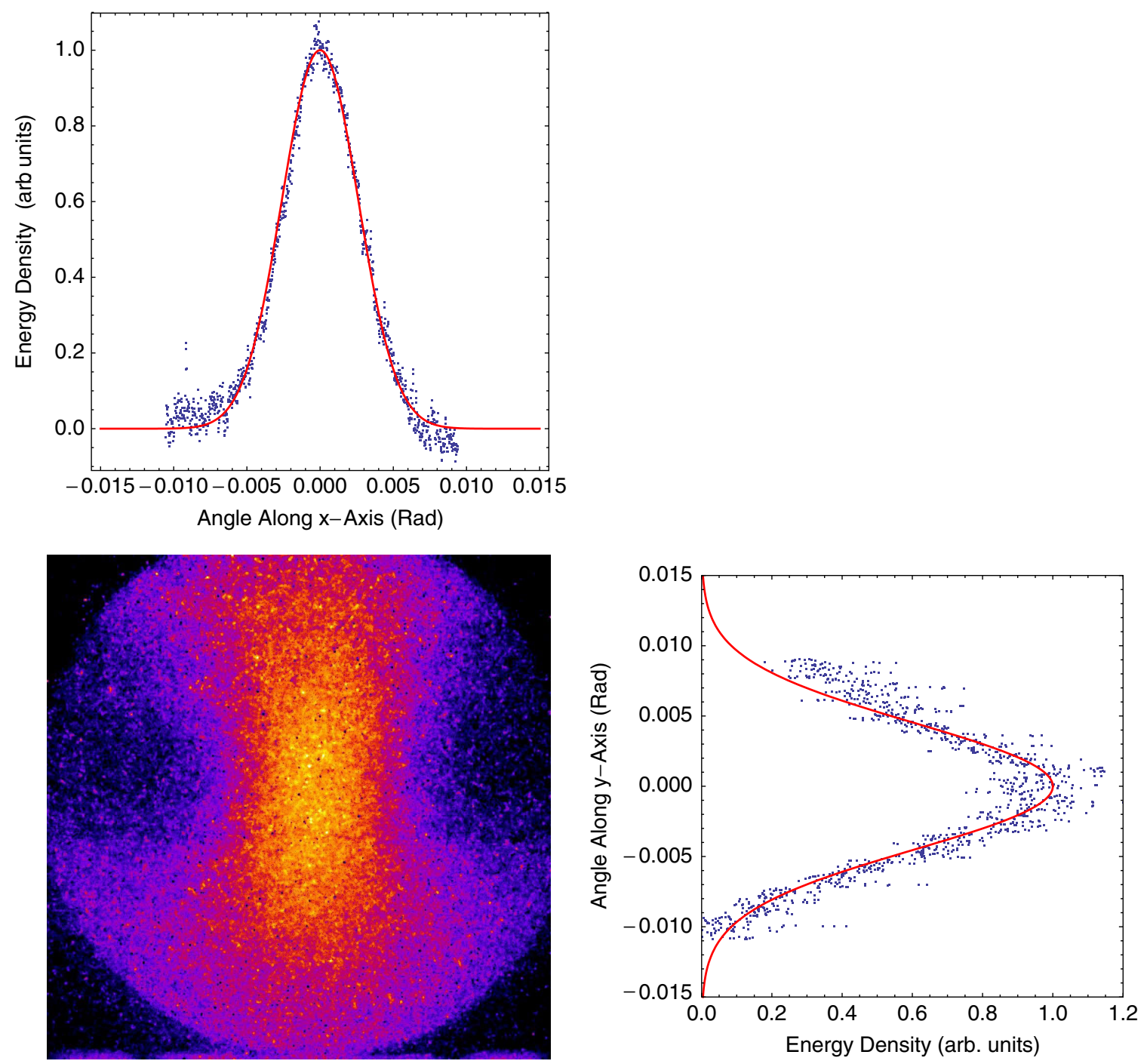

FIG. 4. (Color) False color image of the $\gamma$ rays recorded by the ICCD over $15 \mathrm{~s}$ of integration at $10 \mathrm{~Hz}$ and $2 \mathrm{~m}$ away from the interaction region. On the bottom and left of the image, the angular energy distribution are displayed for the horizontal $(x)$ and vertical (y) axis, respectively. The solid curves represent the Gaussian fit of the experimental data points (dots).

interaction region. The divergence is lower along the horizontal direction due to polarization effects [26]. The full width half maximum (FWHM) of the beam is $6.0 \mathrm{mrad}$ and $10.4 \mathrm{mrad}$ along the horizontal $(x)$ and vertical $(y)$ axis, respectively. By integrating the total counts from the beam profile in Fig. 4, one obtains a total number of $1.6 \times$ $10^{5}$ photons/shot.

\section{2. $\gamma$-ray intensity as a function of delay between laser and electron beams}

When the laser and electron beams are collinear, varying the delay is equivalent to a change in the laser spot size from its minimum waist $w_{0}$ and photon density at the interaction point. Thus, the $\gamma$-ray intensity as a function of the delay between the two beams varies as a Lorentzian $1 /\left[1+\left(z / z_{0}\right)^{2}\right]$, where $z_{0}=\pi w_{0}^{2} / \lambda_{0}$ is the Rayleigh length. Since the beta function of the electron beam focusing line is longer than the Rayleigh length in our geometry, the $\gamma$-ray intensity variation is dominated by the change in the laser spot size. We have used an optical delay line to vary the timing between the laser pulse and the electron bunch, which allowed us to measure the $\gamma$-ray intensity change, averaged over 25 shots, displayed on Fig. 5. The experimental curve can be fitted by a Lorentzian of width $\Delta t=25 \mathrm{ps}$, which corresponds to a Rayleigh length $z_{0}=$ $c \Delta t=7.5 \mathrm{~mm}$. The focal spot inferred from this measurement is $29 \mu \mathrm{m}$ (FWHM) at $\lambda_{0}=532 \mathrm{~nm}$. This value is 2.5 times smaller than the actual FWHM focal spot size $\left(w_{0}=\right.$ $75 \mu \mathrm{m}$ ), indicating a nondiffraction limited laser opera- 


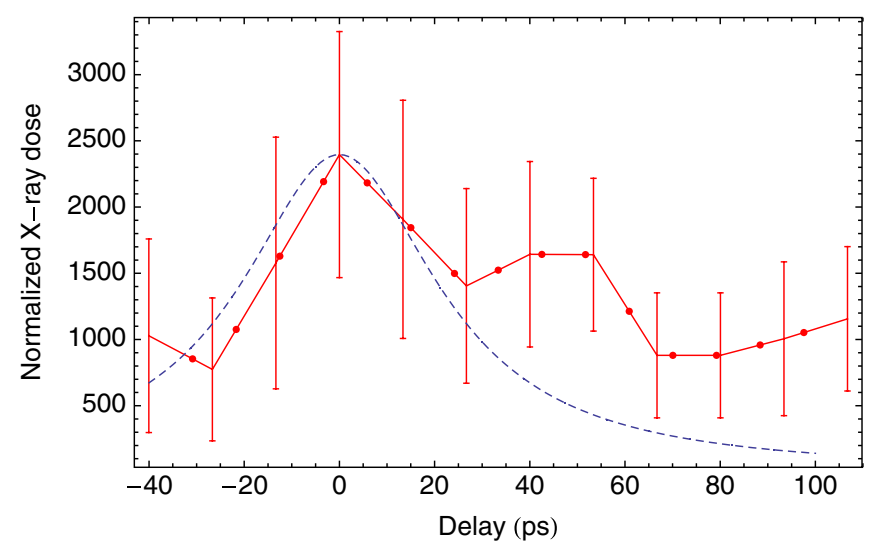

FIG. 5. (Color) Experimentally measured variation of the $\gamma$-ray intensity as a function of the delay $t$ between the laser and the $\gamma$-ray beams (dots) and Lorentzian fit $1 /\left[1+(t / \Delta t)^{2}\right]$, where $\Delta t=25 \mathrm{ps}$ (dashed curve). The error bars are the standard deviation of the intensity averaged over 25 shots.

tion. On Fig. 5 one can observe a ripple for delays larger than 40 ps that deviates from the theoretical Lorentzian shape. It is explained by the fact that the laser pulse was not optimally compressed. Indeed, we recorded autocorrelation traces of the compressed pulse, which indicated that only $\sim 20 \%$ of the total energy (at $1064 \mathrm{~nm}$ ) was within $16 \mathrm{ps}$ and that the rest was contained in wings on each side of the compressed pulse.

\section{B. Spectral measurements}

\section{On-axis spectra}

In Fig. 6, the spectrum of on-axis photons is shown. To faithfully measure the on-axis spectrum, we have used a $6 \mathrm{~mm}$ diameter lead collimator to aperture the beam, placed 3 meters away from the interaction point. The collimator was aligned to the center of the spatial image, as shown in the inset of Fig. 6. The spectrum displayed in

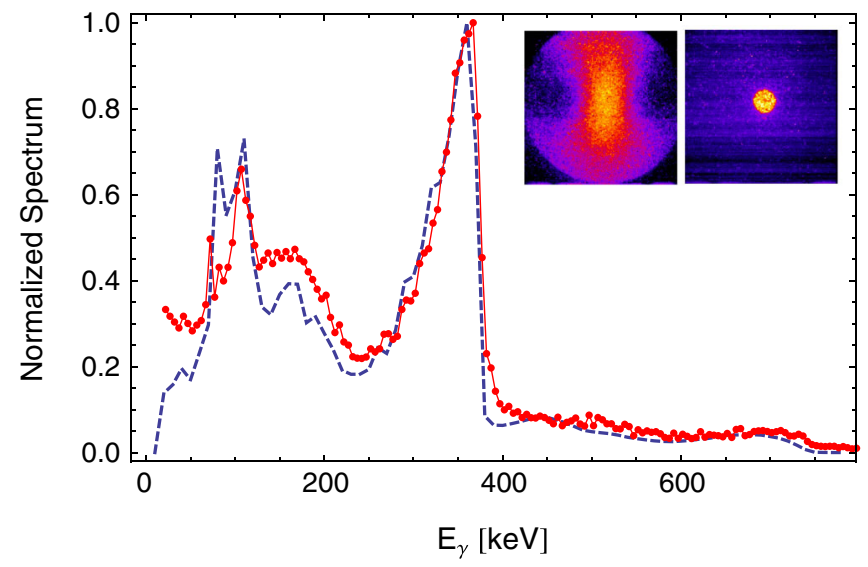

FIG. 6. (Color) On-axis spectrum recorded after scattering off the $\mathrm{Al}$ plate and corresponding Monte Carlo simulation. The images correspond to the full beam and the signal transmitted through the collimator, respectively.
Fig. 6 corresponds to $5 \mathrm{keV}$ bins and to 6.5 hours of data recorded at $10 \mathrm{~Hz}$. The measured spectrum is compared with the simulated pulse height spectrum expected for single-photon counting.

As can be seen in Fig. 6, the spectrum has several distinctive features. The tail after $400 \mathrm{keV}$ is mainly due to the high-energy bremsstrahlung and to pileup (multiplephoton events) in the detector. The main peak has a maximum for $365 \mathrm{keV}$, which corresponds to an incident energy of $478 \mathrm{keV}$. The FWHM of this peak is $55 \mathrm{keV}$ (within limitation of $5 \mathrm{keV}$ bins), which corresponds to a relative bandwidth of $15 \%$. By differentiating (20), it corresponds to source (at the aluminum plate) bandwidth of $12 \%$. The narrower energy spectra (on the order of $1 \%$ reported in Ref. [18]) have been computed with different parameters that were not the measured experimental parameters in our case. In particular, the bandwidth of the gamma-ray spectrum increases as the square of the normalized emittance, which is the most critical point for the design of a narrow band Compton-scattering source. The energy spread and jitter of the laser are negligible, while those of the electron beam are $0.2 \%$ and $j=2$, respectively. This means that the broadening effect on the gamma rays is, using Eq. (1), $0.4 \%$ from the electron beam energy spread. It is much smaller than the broadening effect $(4.5 \%)$ due to the electron beam divergence $\epsilon / \gamma r$, where $r$ is the electron beam radius. The fact that we obtained a bandwidth of $12 \%$ can also be explained by nonlinear broadening effects and by the electron beam position and angular jitter, since we are not showing a single shot but an average (over several hours) spectrum in Fig. 6.

At energies lower than $250 \mathrm{keV}$, a broad continuum can be seen, on which two other peaks (respectively located around 80 and $110 \mathrm{keV}$ ) are superposed. Those are not physical features of the source itself but are the result of several interaction processes, which can be very well tracked and reproduced by Monte Carlo simulations. For modeling we used the MCNP5 code [27], with modifications to include Compton scattering of linearly polarized photons [28]. The $\gamma$-ray source spectrum was calculated using the MATHEMATICA script described in Sec. II, and used by MCNP5 to sample the energy of each source photon in the incident pencil beam $(5 \mathrm{~cm}$ diameter). The polarization was assumed to be $100 \%$ in the horizontal plane (including the beam axis and HPGe detector). All the major components of the experimental setup, such as walls, air, and lead shielding were implemented in the MCNP5 geometry description. Figure 6 shows the simulated pulse height spectrum expected for single-photon counting.

The continuum below $250 \mathrm{keV}$ is due to incomplete energy absorption (elastic Compton scattering) in the detector itself. The broad peak at $110 \mathrm{keV}$ arises from double Compton scattering off the Al plate and adjacent wall, followed by photoabsorption in the Ge detector. Since the detector is shielded with lead, the first peak is due to $\mathrm{x}$ rays 
coming from the lead $\mathrm{K}_{\alpha}$ and $\mathrm{K}_{\beta}$ lines, respectively, at $72.8,75,84.9$, and $87.3 \mathrm{keV}$. The $5 \mathrm{keV}$ binning applied to the spectrum does not allow us to distinguish those lines.

\section{Off-axis spectra: energy-angle correlation}

The energies of $\gamma$ rays produced by Compton scattering vary within the cone of radiation. According to (1), the highest $\gamma$-ray energy $4 \gamma^{2} E_{L}$ is on-axis and the off-axis $\gamma$-ray energies should be lower. To verify this characteristic property of Compton scattering we have measured the spectrum off-axis, at two different positions, for an electron beam energy of $125 \mathrm{MeV}$ and a laser wavelength of $532 \mathrm{~nm}$. To do so, we have used the same $6 \mathrm{~mm}$ lead aperture as for the on-axis measurement, and to alter the direction of the $\gamma$-ray beam, the incident electron beam was deflected with two small steering magnets, so that the position of the electrons at the interaction point was fixed, while the beam angle could be varied by several milliradians. It was not possible to move the collimator instead as it is precisely pointing toward the shielded room where the detectors are. Our measurements correspond to a $3 \mathrm{mrad}$ off-axis measurement in the upper part of the beam and to a
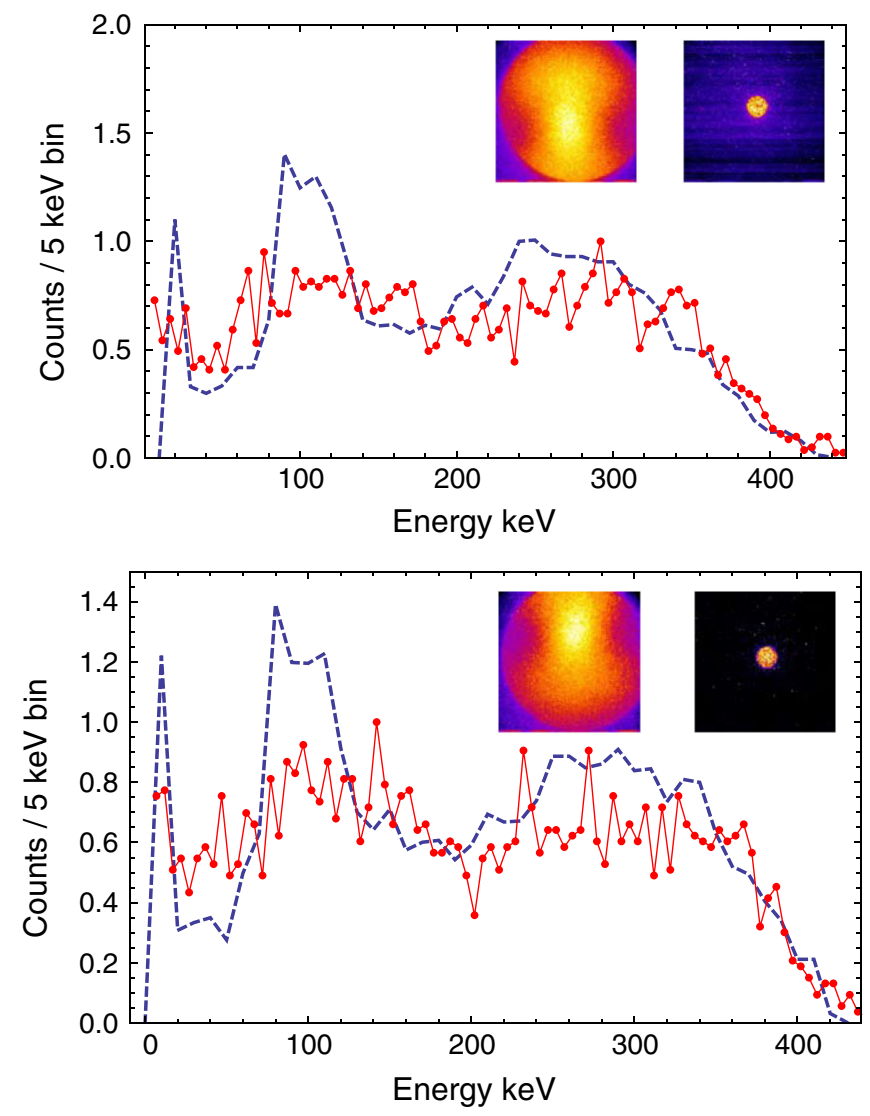

FIG. 7. (Color) Off-axis spectra measured (dots) for the upper lobe ( $3 \mathrm{mrad}$ away from the center) and the lower lobe $(2.5 \mathrm{mrad}$ away from the center), with the corresponding Monte Carlo simulations (dashed curves).
$2.5 \mathrm{mrad}$ off-axis measurement in the lower part of the beam, as shown in Fig. 7. As expected, for both lobes the spectrum is broader than on-axis, with a peak at $\sim 250 \mathrm{keV}$ for the upper lobe and a peak at $\sim 290 \mathrm{keV}$ for the lower lobe as seen on the plots resulting from the Monte Carlo simulation with MCNP5. The parameters of the simulation are the same as for the on-axis spectrum calculation except for the angle of observation that has been changed according to the upper and lower part of the beam.

\section{Energy scaling with the electron beam energy}

On axis, the Compton scattered $\gamma$-ray energy theoretically scales with $4 \gamma^{2}$ for a given laser photon energy (if we neglect the recoil), which we verified by tuning the electron beam to four different energies: $116,101,85$, and $68 \mathrm{MeV}$, and by measuring the spectrum for each case. Identical alignment of the collimator with the center of the beam has been maintained throughout the measurement. The four spectra are displayed in the top part of Fig. 8, where each data set corresponds to $1 \mathrm{~h}$ of acquisition at $10 \mathrm{~Hz}$ and in a single photon-counting mode (rate of $20 \%$ ). The $116 \mathrm{MeV}$ data correspond to 6.5 hours of acquisition and has been
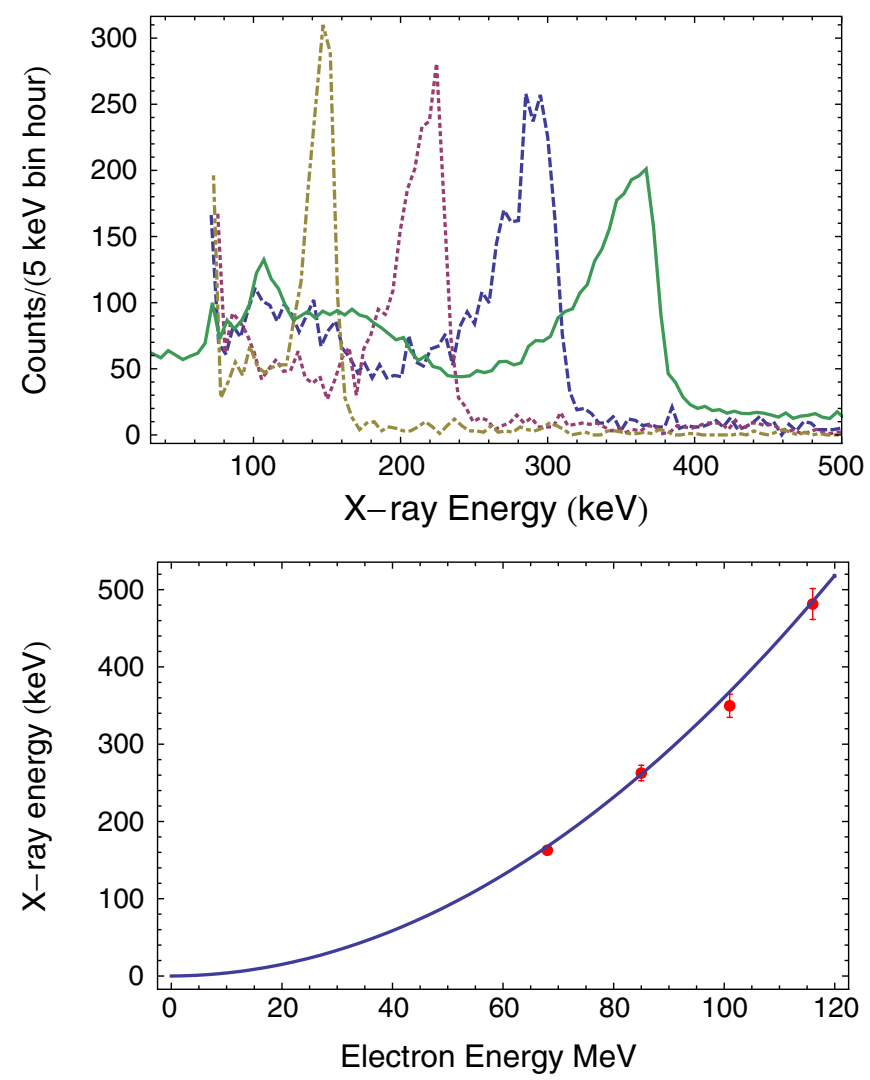

FIG. 8. (Color) Top: Experimentally measured on-axis spectra for electron beam energies of $68 \mathrm{MeV}$ (dot-dashed line), $85 \mathrm{MeV}$ (dotted line), $101 \mathrm{MeV}$ (dashed line), and $116 \mathrm{MeV}$ (solid line). Bottom: experimentally measured peak $\gamma$-ray energy points versus $\gamma$ and theoretical peak $\gamma$-ray energy $E_{x}=4 \gamma^{2} E_{L}$. 
normalized to be within the same amplitude as the other plots. From this measurement, the incident peak $\gamma$-ray energy on the Al plate can be retrieved by using (20). The bottom part of Fig. 8 displays the incident peak $\gamma$-ray energy (inferred from the experimental data) versus the electron relativistic factor $\gamma$. On top of the data points is plotted the theoretical expected $\gamma$-ray energy $4 \gamma^{2} E_{L}$, where $E_{L}=2.33 \mathrm{eV}$ is the laser energy. This shows good agreement and validates the $4 \gamma^{2}$ signature scaling law of laser-based Compton scattering for our source.

\section{High-energy bremsstrahlung}

The linac itself is a source of high-energy $\gamma$ rays because it is well known that the electromagnetic field in a high gradient rf structure can cause electron emission from the copper walls of the accelerating sections. Those electrons can then be accelerated by the fields and interact with metal present in the linac, which potentially yields high-energy $\gamma$ rays on axis. The dark current can reduce the signal to noise ratio for the Compton scattered $\gamma$ rays. To measure this background, we placed one HPGe directly in the incident beam. During this measurement, only the rf power of the linac was enabled while all the lasers (ILS and PDL) were turned off. In addition, to avoid saturation on the detector, we placed 5 inches of lead in the beam path to reduce the count rate to 0.2 /pulse. Since low energy x rays are highly attenuated by the lead absorber, only bremsstrahlung arising from the dark current was measured. The resulting spectrum is presented in Fig. 9, on which the $0.511 \mathrm{MeV}$ line from electron-positron annihilation pairs can well be seen. The incident number of $\gamma$-ray photons on the detector can be simply retrieved by taking into account the attenuation through 5 inches of lead (NIST-XCom database) and the efficiency of the detector (50\%). At $1 \mathrm{MeV}$, one finds 1.1 photons $/ 0.1 \% \mathrm{BW} /$ shot from bremsstrahlung.

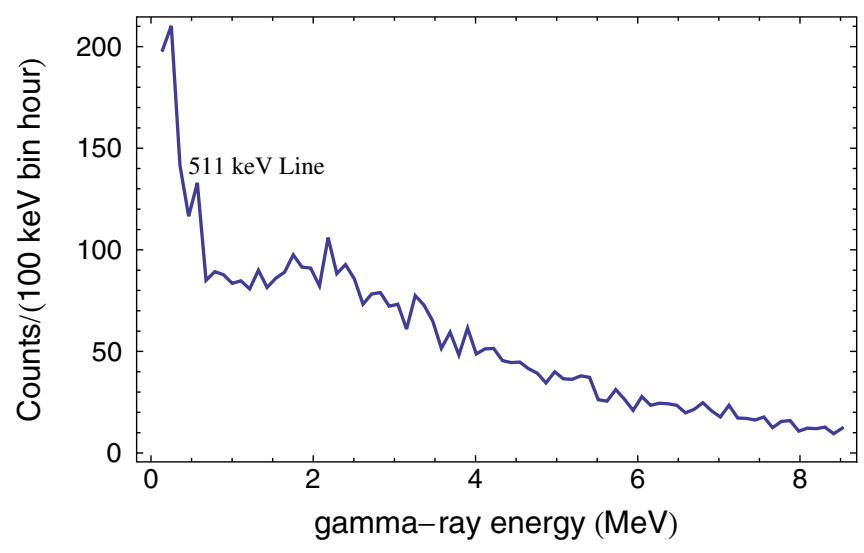

FIG. 9. (Color) High-energy bremsstrahlung arising from the linac dark current measured between 0 and $8 \mathrm{MeV}(100 \mathrm{keV}$ bins) and behind 5 inches of lead.

\section{Summary: On-axis peak brilliance}

With the experimental results obtained throughout the experiments, one can compute the on-axis peak brilliance of the source. The only parameter that has not been directly measured during this experimental campaign is the Compton gamma-ray source size. However, it can be inferred from the overlap of the electron beam and laser beam focal spots, which have been measured at $40 \mu \mathrm{m}$ (rms) and $34 \mu \mathrm{m}$ (rms), respectively. With a spectral bandwidth of $12 \%$ at $478 \mathrm{keV}$, a pulse duration of $16 \mathrm{ps}$ (FWHM, inferred from the laser and electron beam durations), a dose of $1.6 \times 10^{5}$ photons/shot, and a divergence of $10.4 \times 6 \mathrm{mrad}^{2}(\mathrm{FWHM})$, one obtains an on-axis peak brilliance of $1.5 \times 10^{15}$ photons $/ \mathrm{mm}^{2} / \mathrm{mrad}^{2} / \mathrm{s} / 0.1 \%$ bandwidth, which is roughly the brilliance of the APS synchrotron at this energy, currently the brightest synchrotron in the United States [5]. However, since the main goal of this experiment was the proof of principle demonstration of NRF, neither the laser nor the electron beam were fully optimized. However, by using the code described in this paper and that has been benchmarked against our experimental results, we can predict a peak brilliance on the order of $1 \times 10^{21}$ photons $/ \mathrm{mm}^{2} / \mathrm{mrad}^{2} / \mathrm{s} / 0.1 \%$ bandwidth at $478 \mathrm{keV}$ by using state of the art parameters for the electron ( $1 \mathrm{nC}, 1 \mathrm{~mm} \mathrm{mrad})$ and laser $(1 \mathrm{~J})$ beams, as shown in Fig. 10, with detailed parameters specified in Table II. This is 6 orders of magnitude higher than the brightness we currently achieved. Several reasons can explain why we did not reach state of the art parameters in the present source:

The linac we used is 40 years old, and the radiofrequency (rf) power is asymmetrically fed to the sections,

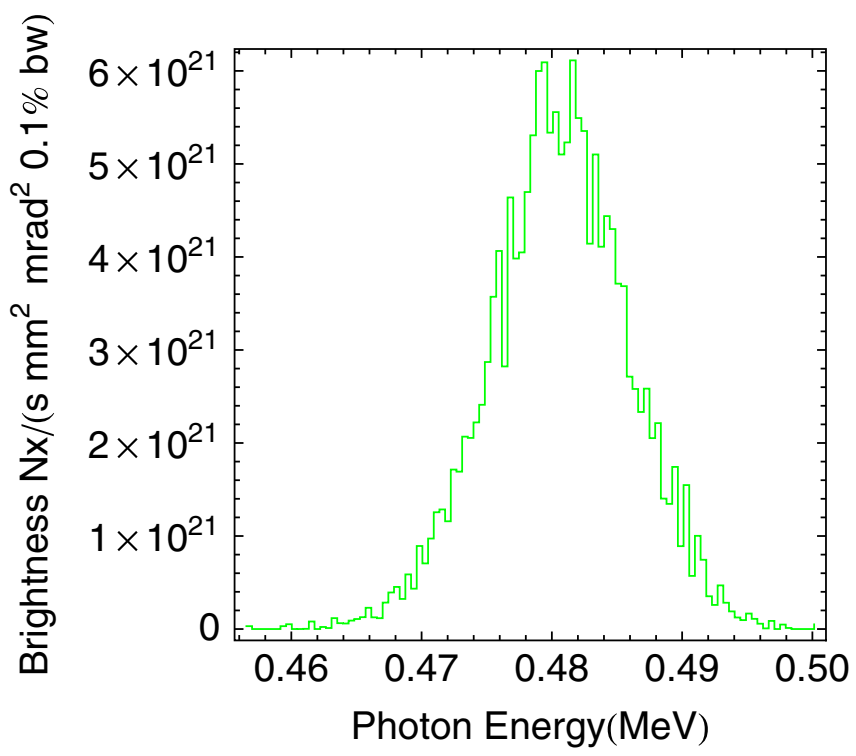

FIG. 10. (Color) On-axis spectrum calculated with a $1 \mathrm{nC}, 1 \mathrm{~mm}$ mrad emittance and $9 \mu \mathrm{m}$ electron beam spot size (rms) and a $1 \mathrm{~J}, 9 \mu \mathrm{m}$ rms size laser spot and no jitter. 
TABLE II. State of the art laser and electron beam parameters used for the brightness calculation.

\begin{tabular}{lc}
\hline \hline Parameter & Specification \\
\hline Laser pulse duration & $10 \mathrm{ps}(\mathrm{FWHM})$ \\
Laser wavelength & $532 \mathrm{~nm}$ \\
Laser spot size & $9 \mu \mathrm{m}(\mathrm{rms}), 100 \%$ energy in spot \\
Laser energy & $1 \mathrm{~J}, 100 \%$ compressed \\
Electron energy & $116 \mathrm{MeV}$ \\
Electron beam spot size & $9 \mu \mathrm{m}(\mathrm{rms})$ \\
Electron bunch length & $10 \mathrm{ps}(\mathrm{FWHM})$ \\
Electron beam charge & $1 \mathrm{nC}$ \\
Normalized emittance & $1 \mathrm{~mm} \mathrm{mrad}$ \\
Jitter factor & 1 \\
\hline \hline
\end{tabular}

which induces emittance growth as the electrons are accelerated.

The laser compression was not optimized, and therefore, all the energy was not contained in the compressed pulse. We used a chirped fiber Bragg grating stretcher, which is very sensitive to temperature variations, and a bulk grating compressor. Because the bandwidths involved here are extremely narrow for chirped pulse amplification, the compressor path length had to be very long $(30 \mathrm{~m})$. We estimate the infrared short pulse to contain only $20 \%$ of the total energy.

Because of the above reason, the nonlinear conversion from infrared to green light was relatively poor. Those technological bottlenecks are being addressed for the design of the next monoenergetic gamma-ray (MEGa-ray) source at LLNL.

The focal laser sport size, measured to be $34 \mu \mathrm{m}$ (rms), did not contain all the energy as well, as shown on beam profile measurements. We had at most $20 \%$ of the total laser energy as well.

\section{APPLICATION: DETECTION OF NUCLEAR RESONANCE FLUORESCENCE IN ${ }^{7} \mathrm{~L}$ I}

\section{A. NRF detection setup}

In addition to measuring the properties of the source, we have used it to detect the NRF line of ${ }^{7} \mathrm{Li}$ at $478 \mathrm{keV}$. For this, we used a $8-\mathrm{cm}$ diameter plastic bottle containing $225 \mathrm{~g}$ of LiH with a density of $0.36 \mathrm{~g} / \mathrm{cm}^{3}$. The sample was located in the shielded $0^{\circ}$ cave, about 20 meters away from the source which is still collimated by the 6-mm lead aperture. When the beam interacts with the lithium, its diameter is on the order of $4 \mathrm{~cm}$. The NRF scattered photons from the ${ }^{7} \mathrm{Li}$ are detected by a second HPGe, similar in size, resolution, and efficiency to the one used for the spectral measurements. As depicted in Fig. 11, the detector is placed at $90^{\circ}$ with respect to the incident beam axis, $15 \mathrm{~cm}$ away from the center of the beam. As only $7 \%$ of the incident $\gamma$ rays are attenuated by the Al plate, we have kept the spectral diagnostic in order to verify the proper tuning of the beam throughout the measurement.

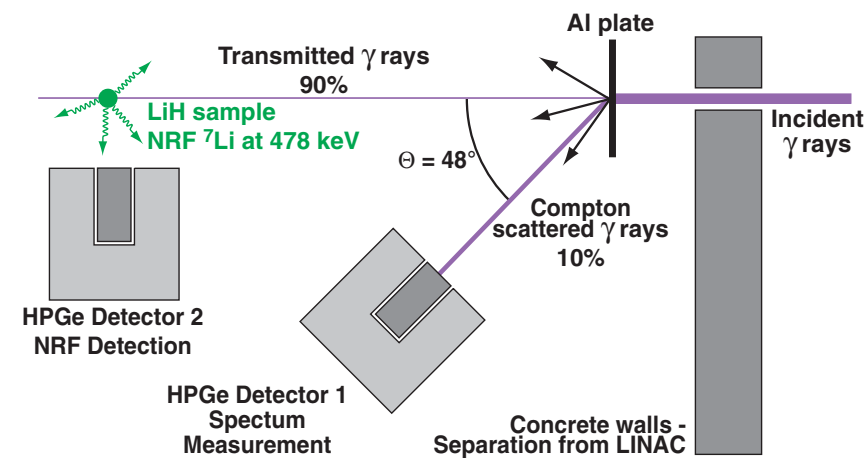

FIG. 11. (Color) NRF detection setup.

With the $\gamma$-ray production described above at $478 \mathrm{keV}$, NRF photons were expected to be scattered isotropically at a rate of 16 photons/hour. The HPGe detector was positioned in the $\mathrm{x}$-ray polarization plane to maximize the NRF signal from the M1 transition in ${ }^{7} \mathrm{Li}$ [29]. A scattering angle of $90^{\circ}$ was chosen in order to minimize the amount of Compton scatter background from the LiH target [30]. A $1 \mathrm{~cm}$ thick $\mathrm{Pb}$ absorber in front of the HPGe detector reduced the count rate to $10 \%$. It also serves as a demonstration that detecting low- $Z$ and low-density material behind high- $Z$, high-density material is feasible with this process. Indirect detection methods have also been successfully employed with this source [31]

\section{B. NRF results}

NRF data have been acquired for 7.5 hours at a $10 \mathrm{~Hz}$ repetition rate and the full spectrum obtained on the detector between 0 and $1.6 \mathrm{MeV}$ is displayed in Fig. 12 on a logarithmic scale. Besides the $478 \mathrm{keV}$ NRF line from ${ }^{7} \mathrm{Li}$, several distinguishable features can be observed on this plot. The continuum centered around $250 \mathrm{keV}$ corresponds to Compton scattering of the $478 \mathrm{keV}$ radiation off the $\mathrm{LiH}$ sample at an angle of $90^{\circ}$. As in the source spectrum, the peaks corresponding to the lead fluorescence around $80 \mathrm{keV}$ are present. The line at $511 \mathrm{keV}$ results from the

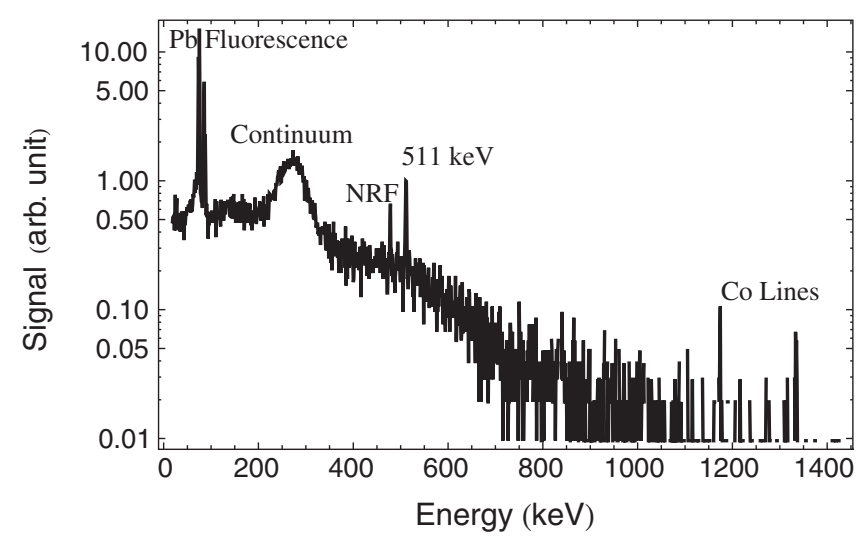

FIG. 12. NRF spectrum and features. 


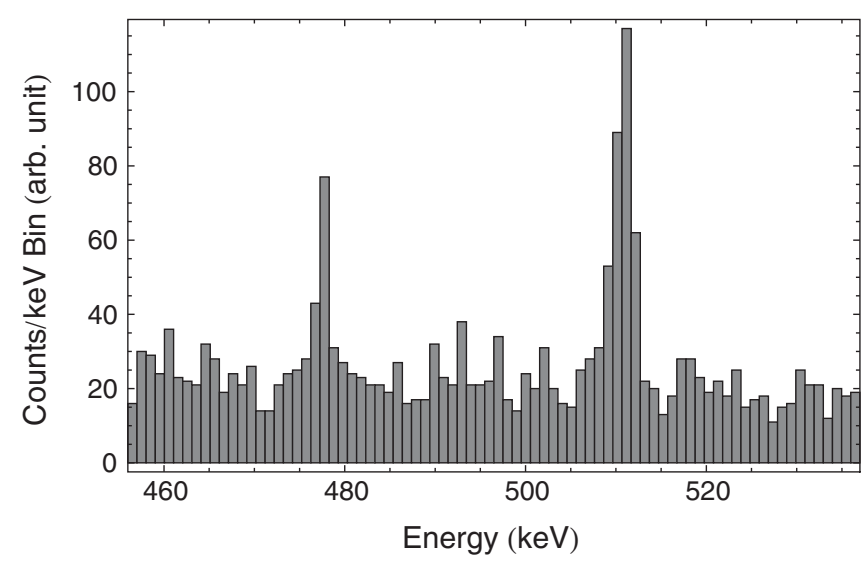

FIG. 13. (Color) NRF scattered spectrum between 460 and $540 \mathrm{keV}$, showing the NRF line with a $6 \sigma$ confidence level and the $511 \mathrm{keV}$ line.

$e^{+}-e^{-}$annihilation pairs created by the high-energy bremsstrahlung from the linac (this line remains present when we block the ILS). The lines at 1.17 and $1.33 \mathrm{MeV}$ arise from ${ }^{60} \mathrm{Co}$ activation naturally present in the cave walls.

A closer look at the scattered spectrum between 460 and $540 \mathrm{keV}$ (Fig. 13), showing the NRF line and the $511 \mathrm{keV}$ line, indicates a detection confidence level of $6 \sigma$, which means that statistically (assuming a normal distribution with standard deviation $\sigma$ ), we are above the background so that the confidence of our measurement is superior to $99.99 \%$. Because the theoretical width of the NRF line from ${ }^{7} \mathrm{Li}(\mathrm{a}$ few $\mathrm{eV}$ ) is much lower than the measured resolution of the germanium detector $(2.8 \mathrm{keV})$, it is not possible to estimate the experimental width of the line.

\section{CONCLUSION AND OUTLOOK}

In conclusion, we have demonstrated and characterized Compton scattering from a novel high-brightness $\gamma$-ray source. This source has been exhaustively studied by a series of different diagnostics, allowing us to measure spectral (12\% bandwidth at $478 \mathrm{keV})$ and spatial data (low divergence), as well as characteristic signatures of the Compton-scattering mechanism $\left(4 \gamma^{2}\right.$ scaling and energy-angle correlation). The key parameter of this source is its peak brilliance, $1.5 \times 10^{15}$ photons $/ \mathrm{mm}^{2} /$ $\operatorname{mrad}^{2} / \mathrm{s} / 0.1 \%$ bandwidth at $\mathrm{MeV}$-range energies. As it scales with the square of the electron beam relativistic factor divided by its normalized emittance, it will be enhanced at higher $\gamma$-ray energies by pursuing the development of new technologies associated with this source. High acceleration gradient $\mathrm{x}$-band linac systems, robust and high-average power fiber laser systems are all part of the design of a new MEGa-ray precision machine currently developed at LLNL. In this paper, we have demonstrated, by detecting nuclear resonance fluorescence from ${ }^{7} \mathrm{Li}$ at $478 \mathrm{keV}$, that this new class of Compton-scattering sources will have tremendous applications in nuclear photoscience.

\section{ACKNOWLEDGMENTS}

This work performed under the auspices of the U.S. Department of Energy by Lawrence Livermore National Laboratory under Contract No. DE-AC52-07NA27344. The authors wish to thank Gerry Anderson for the electron beam operations and detectors shielding, and Shawn Betts for laser operations.

[1] R. Schoenlein, W. Leemans, A. Chin, P. Volfbeyn, T. Glover, P. Balling, M. Zolotorev, K. Kim, S. Chattopadhyay, and C. Shank, Science 274, 236 (1996).

[2] A. Rousse, C. Rischel, and J-C. Gauthier, Rev. Mod. Phys. 73, 17 (2001).

[3] F. Zernike, Science 121, 345 (1955).

[4] E. Roessl and R. Proksa, Phys. Med. Biol. 52, 4679 (2007).

[5] D. Attwood, Soft X-rays and Extreme Ultraviolet Radiation (Cambridge University Press, Cambridge, England, 1999), Chap. 5.

[6] http://ssrl.slac.stanford.edu/lcls/.

[7] http://www.xfel.eu/.

[8] U. Kneissl, H. M. Pitz, and A. Zilges, Prog. Part. Nucl. Phys. 37, 349 (1996).

[9] P. Coleman, Positron Beams and Their Applications (World Scientific, Singapore, 2000).

[10] H. R. Weller, M. W. Ahmed, H. Gao, W. Tornow, Y. K. Wu, M. Gai, and R. Miskimen, Prog. Part. Nucl. Phys. 62, 257 (2009).

[11] V. N. Litvinenko, Nucl. Instrum. Methods Phys. Res., Sect. A 507, 527 (2003).

[12] F. V. Hartemann, H. A. Baldis, A. K. Kerman, A. Le Foll, N. C. Luhmann, Jr., and B. Rupp, Phys. Rev. E 64, 016501 (2001).

[13] W. P. Leemans, R. V. Schoenlein, P. Volfbeyn, A. H. Chin, T.E. Glover, P. Balling, M. Zolotorev, K-J. Kim, S. Chattopadhyay, and C.V. Shank, IEEE J. Quantum Electron. 33, 1925 (1997).

[14] S. K. Ride, E. Esarey, and M. Baine, Phys. Rev. E 52, 5425 (1995).

[15] F. V. Hartemann and A. K. Kerman, Phys. Rev. Lett. 76, 624 (1996).

[16] E. Esarey, S. K. Ride, and P. Sprangle, Phys. Rev. E 48, 3003 (1993).

[17] F. V. Hartemann, High Field Electrodynamics (CRC Press, Boca Raton, FL, 2002).

[18] F. V. Hartemann, W. J. Brown, D. J. Gibson, S. G. Anderson, A. M. Tremaine, P. T. Springer, A. J. Wootton, E. P. Hartouni, and C. P. J. Barty, Phys. Rev. ST Accel. Beams 8, 100702 (2005).

[19] G. Bhatt, H. Grotch, E. Kazes, and D. A. Owen, Phys. Rev. A 28, 2195 (1983).

[20] D. J. Gibson, S. G. Anderson, C. P. J. Barty, S. M. Betts, R. Booth, W. J. Brown, J. K. Crane, R. R. Cross, D. N. Fittinghoff, F. V. Hartemann, J. Kuba, G. P. Lesage, D. R. Slaughter, A. M. Tremaine, A. J. Wooton, E. P. Hartouni, P. T. Springer, and J. B. Rosenzweig, Phys. Plasmas 11, 2857 (2004). 
[21] W. J. Brown, S. G. Anderson, C. P. J. Barty, S. M. Betts, R. Booth, J. K. Crane, R. R. Cross, D. N. Fittinghoff, D. J. Gibson, F. V. Hartemann, E. P. Hartouni, J. Kuba, G. P. Le Sage, D. R. Slaughter, A. M. Tremaine, A. J. Wooton, and P. T. Springer, Phys. Rev. ST Accel. Beams 7, 060702 (2004).

[22] F. V. Hartemann, A. M. Tremaine, S. G. Anderson, C. P. J. Barty, S. M. Betts, R. Booth, W. J. Brown, J. K. Crane, R. R. Cross, D. J. Gibson, D. N. Fittinghoff, J. Kuba, G. P. Le Sage, D. R. Slaughter, A. J. Wootton, E. P. Hartouni, P. T. Springer, J. B. Rosenzweig, and A. K. Kerman, Laser Part. Beams 22, 221 (2004).

[23] D. J. Gibson et al., preceding article, Phys. Rev. ST Accel. Beams 13, 070703 (2010).

[24] D. Strickland and G. Mourou, Opt. Commun. 56, 219 (1985).
[25] http://physics.nist.gov/PhysRefData/Xcom/Text/ XCOM.html.

[26] W. J. Brown and F. V. Hartemann, Phys. Rev. ST Accel. Beams 7, 060703 (2004).

[27] R. A. Forster et al., Nucl. Instrum. Methods Phys. Res., Sect. B 213, 82 (2004).

[28] G. Matt, M. Feroci, M. Rapisarda, and E. Costa, Radiat. Phys. Chem. 48, 403.

[29] N. Pietralla et al., Phys. Rev. Lett. 88, 012502 (2001).

[30] L. W. Fagg and S. S. Hanna, Rev. Mod. Phys. 31, 711 (1959).

[31] F. Albert, S. G. Anderson, G. A. Anderson, S. M. Betts, D. G. Gibson, C. A. Hagmann, J. Hall, M. S. Johnson, M. J. Messerly, V. A. Semenov, M. Y. Shverdin, A. M. Tremaine, F. V. Hartemann, C. W. Siders, D. P. McNabb, and C. P. J. Barty, Opt. Lett. 35, 354 (2010). 